\title{
Experiencia operatoria de los residentes de cirugía general: Resultados de un estudio transversal multinstitucional en Colombia
}

\author{
Operative experience of general surgery residents: \\ Results of a cross-sectional multinstitutional study in Colombia
}

Sebastián Sierra-Sierra ${ }^{\mathbb{D}}$, Luis Carlos Domínguez² $\mathbb{D}$, Lilian Torregrosa-Almonacid ${ }^{3} \mathbb{D}$

1 Médico, especialista en Cirugía General; profesor de Cirugía, Universidad de Antioquia, Medellín, Colombia.

2 Médico, especialista en Cirugía General; profesor asociado, Departamento de Cirugía, Universidad de la Sabana, Chía, Colombia.

3 MD, FACS, especialista en Cirugía general, Cirugía de Seno y Tejidos Blandos y magíster en Bioética; profesora asociada de Cirugía, Directora Departamento de Cirugía y Especialidades, Facultad de Medicina, Pontificia Universidad Javeriana, Hospital Universitario San Ignacio. Presidente, Asociación Colombiana de Cirugía, Bogotá, D.C., Colombia.

\section{Resumen}

Introducción. Una parte fundamental del entrenamiento de un residente de cirugía es lograr un volumen operatorio suficiente para alcanzar una competencia adecuada en su vida laboral independiente. Tanto el volumen como la autonomía, son desafíos para los programas de residencia en cirugía general de Colombia.

Métodos. Se realizó un estudio multinstitucional, con la participación de 5 programas de especialización en cirugía general, de diferentes regiones del país. Se utilizó la bitácora electrónica Logbook para el registro de procedimientos durante un periodo de 12 meses. Se hizo un análisis de la base de datos recolectada.

Resultados. Un total de 111 médicos residentes participaron en el estudio. Se registraron 29.622 procedimientos quirúrgicos, que correspondieron a 23.206 pacientes. El 51,7 \% de los procedimientos fueron cirugías electivas, el $46,9 \%$ cirugías de urgencia y el $1 \%$ de emergencia. El 22,6 \% de los procedimientos se realizó a través de un abordaje mínimamente invasivo. Los cinco procedimientos quirúrgicos más frecuentemente registrados fueron: colecistectomía ( $n=4341)$, apendicectomía $(n=2558)$, herniorrafia inguinal $(n=2059)$, herniorrafia umbilical $(n=1225)$ y lavado peritoneal $(\mathrm{n}=1198)$. En promedio, cada residente realizó 27 cirugías por mes y en estos procedimientos el rol predominante del residente fue el de cirujano principal, desde momentos tempranos en su formación (a partir del segundo año).

Discusión. Es posible lograr a nivel nacional el registro de las actividades a través de una bitácora. Los médicos residentes colombianos realizan un número de cirugías similares o mayores a lo descrito en otros países. Debemos actualizar nuestras expectativas según la formación que reciben actualmente los médicos residentes.

Palabras clave: educación médica; programas de postgrado; cirugía general; educación basada en competencias; sistema de registros; registros electrónicos; Colombia.

Fecha de recibido: 13/01/2021 - Fecha de aceptación: 24/01/2021 - Fecha de publicación en línea: 29/03/2021

Correspondencia: Sebastian Sierra Sierra, Dirección: Clínica CES. Calle 58 N 50C-2, Medellín, Colombia. Teléfono: (57) (4) 5767373. Correo electrónico: sebastiancirugia@gmail.com

Citar como: Sierra-Sierra S, Domínguez LC, Torregrosa-Almonacid L.Experiencia operatoria de los residentes de cirugía general: Resultados de un estudio transversal multinstitucional en Colombia. Rev Colomb Cir. 2021;36:411-20. https://doi.org/10.30944/20117582.762

Este es un artículo de acceso abierto bajo una Licencia Creative Commons - BY-NC-ND https://creativecommons.org/licenses/by-ncnd/4.0/deed.es 


\begin{abstract}
Introduction. A critical part of training for a surgical resident is achieving sufficient operating volume to achieve adequate competence in their independent practice. Both volume and autonomy are challenges for general surgery residency programs in Colombia.
\end{abstract}

Methods. A multinstitutional study was performed, with the participation of 5 specialization programs in general surgery, from different regions of the country. The electronic Logbook was used to record procedures for a period of 12 months. An analysis of the collected database was made.

Results. A total of 111 resident physicians participated in the study. There were 29,622 surgical procedures registered, corresponding to 23,206 patients. Overall, $51.7 \%$ of the procedures were elective surgeries, $46.9 \%$ were urgent surgeries and $1 \%$ were emergency surgeries. $22.6 \%$ of the procedures were performed through a minimally invasive approach. The five most frequently recorded surgical procedures were: cholecystectomy $(n=4341)$, appendectomy $(n=2558)$, inguinal herniorrhaphy $(n=2059)$, umbilical herniorrhaphy $(n=1225)$, and peritoneal lavage $(n=1198)$. On average, each resident performed 27 surgeries per month, and in these procedures the predominant role of the resident was that of the main surgeon, from early in their training (from the second year on).

Discussion. It is possible to achieve a national record of activities through a log. Colombian resident physicians perform a number of surgeries similar or greater than those described in other countries. We must update our expectations based on the training currently received by resident physicians.

Keywords: medical education; health postgraduate programs; general surgery; competency-based education; registries; electronic records; Colombia.

\section{Introducción}

Una parte fundamental del entrenamiento de un residente de cirugía es lograr un volumen operatorio suficiente, con la adquisición gradual de la autonomía para completar los procedimientos quirúrgicos de manera independiente, a medida que disminuye la supervisión ${ }^{1}$. Tanto el volumen como la autonomía, son desafíos para los programas de residencia en cirugía general ${ }^{2}$. Estos desafíos se pueden explicar, entre otras cosas, por el desarrollo de subespecialidades dedicadas a técnicas quirúrgicas cada vez más complejas, por las políticas de reducción de las horas de trabajo hospitalario y por las preocupaciones crecientes por los resultados centrados en el paciente y la eficiencia organizacional ${ }^{3,4}$. En otras palabras, la exigencia de mayor eficiencia en el quirófano puede entrar en conflicto con los procesos de enseñanza.
En respuesta, los programas de entrenamiento quirúrgico han evolucionado hacia modelos de educación basados en competencias (competency based education), los cuales pretenden garantizar la formación de cirujanos competentes y confiables para la sociedad, dentro de estándares educativos y criterios específicos de experiencia operatoria ${ }^{5-7}$.

Este modelo refleja la evolución de los criterios centrados en el "número mínimo" de procedimientos quirúrgicos en los que el residente debe participar como cirujano principal, hacia criterios integrales de evaluación de la competencia operatoria. Los requerimientos de un mínimo volumen operatorio carecen de información objetiva y de evidencia que dé cuenta de su impacto en el aprendizaje y la competencia profesional ${ }^{8,9}$. Completar un "número mínimo" de procedimientos, por lo tanto, no garantiza necesariamente que un 
cirujano sea técnicamente competente. Por lo tanto, dentro de la evaluación integral de la competencia profesional, en el marco de la educación basada en competencia, las Actividades Profesionales Confiables (APC) han cobrado relevancia en Europa y Norteamérica durante la última década ${ }^{10}$.

En Colombia, la experiencia es similar. La Asociación Colombiana de Facultades de Medicina (ASCOFAME) propuso hace más de dos décadas, un mínimo número de procedimientos quirúrgicos que debe realizar un residente de cirugía antes de graduarse ${ }^{11}$. Entre estos procedimientos se incluyen, por ejemplo, cincuenta toracostomías, cincuenta tiroidectomías, veinte amputaciones, veinte gastrectomías y cincuenta colecistectomías abiertas con colangiografía, entre otros. No obstante, la aceptación de estos requisitos ha sido variable entre los programas del país y la exposición del residente a este "número mínimo de procedimientos" ha sido difícil de alcanzar por diferentes razones, entre ellas la dinámica del sistema de salud, la variabilidad demográfica, los sistemas de contratación y el acceso a tecnología ${ }^{12}$. Adicionalmente, no existen registros publicados en el país que reflejen la experiencia operatoria de un residente de cirugía de acuerdo con los requisitos de ASCOFAME, así como la capacidad organizacional de los programas de residencia para garantizarlos. Igualmente, la mayoría de los programas cuentan con currículos de educación quirúrgica tradicional y no han migrado hacia modelos de educación basada en competencia, centrados en la evaluación rigurosa del desempeño operatorio (por ejemplo, a través de actividades profesionales confiables).

Por otra parte, un problema común que subyace al desafío de los "mínimos" y de la evaluación de la competencia operatoria de los residentes de cirugía en Colombia, tiene que ver con la escasa información sobre la actividad real de los cirujanos, frente a las necesidades demográficas y epidemiológicas del país. En otras palabras, aún se necesita información que dé cuenta de los procedimientos más frecuentes que realizan los cirujanos en Colombia, para adaptar la capacidad de los pro- gramas de residencia a la formación de cirujanos confiables en procedimientos específicos. Algunos proyectos aislados han permitido conocer la frecuencia y tipo de procedimientos que realizan los residentes, así como el nivel de autonomía y supervisión que experimentan ${ }^{13,14}$, pero es escasa e insuficiente la información a nivel nacional, que permita conocer la variabilidad regional de la actividad quirúrgica en cirugía general. Esta información es crucial para que los veinte programas de residencia activos en el país, canalicen sus esfuerzos en la evaluación de la competencia operatoria de los residentes en procedimientos específicos de alta prevalencia. Por otra parte, esta información puede contribuir a definir los límites y alcances de los programas de especialización en cirugía general con respecto a los de segundas especialidades.

El presente estudio, realizado desde la Asociación Colombiana de Cirugía, con la participación de varios programas de residencia colombianos, pretende aportar información con respecto a estos vacíos de conocimiento. Nuestro objetivo es describir el tipo y frecuencia de procedimientos quirúrgicos en los que participan los residentes de cirugía general en Colombia.

\section{Métodos}

\section{Diseño del estudio}

Estudio multinstitucional, de corte transversal, realizado en junio de 2020, sobre una base de datos recolectados en forma prospectiva entre abril de 2019 y marzo de 2020.

\section{Participantes}

El estudio se llevó a cabo en cinco programas de Especialización en Cirugía General en Colombia. Se invitó a participar a 111 médicos residentes de cirugía de todos los niveles de entrenamiento, provenientes de dos universidades públicas y tres privadas, localizadas en tres diferentes ciudades del país, así como a los directores de estos programas. 


\section{Recolección de la información y variables}

La recolección de la información se realizó mediante el uso de una bitácora electrónica para el registro de procedimientos quirúrgicos y se utilizó la plataforma Logbook (www.logbook.com.co).

Esta bitácora recopila información diligenciada por el médico residente al final de cada procedimiento quirúrgico en el que participa. El registro puede realizarse desde cualquier dispositivo con acceso a internet y las principales variables que contiene son:

1. Identificación del paciente

2. Diagnóstico postoperatorio de acuerdo con la Clasificación Internacional de Enfermedades (CIE-10).

3. Ámbito del procedimiento quirúrgico: electivo, urgente y emergente.

4. Rotación actual del residente (por ejemplo: cirugía general, cirugía vascular, etcétera)

5. Año de residencia.

6. Tipo de procedimiento quirúrgico: abierto o mínimamente invasivo.

7. Procedimiento quirúrgico realizado. La plataforma cuenta con un listado predeterminado de más de trescientos procedimientos quirúrgicos entre los cuales el residente puede elegir uno o varios procedimientos por paciente. La plataforma permite la inclusión de procedimientos no predeterminados mediante la opción "otro procedimiento" en texto libre.

8. Rol del residente durante el procedimiento quirúrgico: Cirujano principal, primer ayudante o segundo ayudante.

9. Autoevaluación del residente sobre su desempeño operatorio. Esta autoevaluación se realiza con base en una Escala de Likert (1: pobre desempeño, 5: excelente desempeño).

La información recolectada se almacenó en la base de datos de Logbook y periódicamente se enviaron reportes a las universidades para la revisión por los directores de cada programa de especialización. Adicionalmente, cada residente tuvo acceso a sus registros desde su perfil de usuario.

\section{Análisis estadístico}

La información obtenida se presenta en totales, medias, promedios y porcentajes. Todos los análisis se realizaron en Excel (Microsoft Corp).

Los residentes no estuvieron en un mismo año de residencia durante el periodo de estudio por lo que se encontraron valores atípicos en los datos iniciales que pueden ser fuente de ruido para el análisis. Para eliminar el sesgo al calcular los promedios, se utilizó el método del rango intercuartil que elimina aquellos valores muy superiores al tercer cuartil y los muy inferiores al segundo. Después de aplicarlo al conjunto de datos, se pudo llevar a cabo el cálculo de los promedios mensuales y anuales.

\section{Resultados}

Un total de 111 residentes participaron en el estudio. Entre los participantes, el 72,9 \% registraron al menos cinco procedimientos por mes. Se registraron 29.622 procedimientos quirúrgicos, que corresponden a 23.206 pacientes, considerando que en cada paciente se realizaron varios procedimientos (promedio de 1,3 procedimientos por paciente). El 65,7 \% de los procedimientos fueron realizados durante rotaciones de cirugía general, el $51,7 \%$ de los procedimientos fueron cirugías electivas, el $46,9 \%$ cirugías de urgencia y el $1 \%$ de emergencia. El 22,6\% de los procedimientos se realizó a través de un abordaje mínimamente invasivo (31,4 \% por vía laparoscópica).

Las características de los procedimientos reportados por cada programa participante se presentan en la tabla 1, y la distribución de los procedimientos por tipo de rotación se presenta en la tabla 2 . Los cinco procedimientos quirúrgicos más frecuentemente registrados fueron: colecistectomía $(n=4341)$, apendicectomía $(n=2558)$, herniorrafia inguinal ( $\mathrm{n}=2059)$, herniorrafia umbilical $(\mathrm{n}=1225)$ y lavado peritoneal $(n=1198)$. El porcentaje de estos procedimientos realizados por abordaje mínimamente invasivo fue el siguiente: colecistectomía 
Tabla 1. Procedimientos realizados en los programas participantes.

\begin{tabular}{|c|c|c|c|c|c|}
\hline Programa & 1 & 2 & 3 & 4 & 5 \\
\hline Tipo & Público & Público & Privado & Privado & Privado \\
\hline $\begin{array}{l}\text { Número de residentes } \\
\text { de Cirugía general }\end{array}$ & 27 & 11 & 21 & 36 & 16 \\
\hline Total de registros & 3104 & 4626 & 3353 & 9626 & 2497 \\
\hline $\begin{array}{l}\text { Total de } \\
\text { procedimientos }\end{array}$ & 3954 & 6335 & 3957 & 12369 & 3007 \\
\hline $\begin{array}{l}\text { Número de } \\
\text { procedimientos } \\
\text { electivos }(\%)\end{array}$ & $2436(61,6 \%)$ & $3035(47,9 \%)$ & $1999(50,5 \%)$ & $5704(46,1 \%)$ & $1958(65,1 \%)$ \\
\hline $\begin{array}{l}\text { Número de } \\
\text { procedimientos } \\
\text { de urgencia (\%) }\end{array}$ & $1306(33 \%)$ & $3130(49,4 \%)$ & $1896(47,9 \%)$ & $6561(53 \%)$ & $1004(33,4 \%)$ \\
\hline $\begin{array}{l}\text { Número de } \\
\text { procedimientos } \\
\text { de emergencia (\%) }\end{array}$ & $122(3,1 \%)$ & $170(2,7 \%)$ & $62(1,6 \%)$ & $102(0,8 \%)$ & $45(1,5 \%)$ \\
\hline $\begin{array}{l}\text { Número de } \\
\text { procedimientos } \\
\text { mínimamente } \\
\text { invasivos (\%) }\end{array}$ & $1042(26,4$ \%) & $1067(16,8 \%)$ & $979(24,7 \%)$ & $2528(20,4 \%)$ & $1065(35,4 \%)$ \\
\hline $\begin{array}{l}\text { Promedio de registros } \\
\text { por residente por mes }\end{array}$ & 9,6 & 35 & 13,3 & 22,3 & 13 \\
\hline $\begin{array}{l}\text { Promedio de } \\
\text { procedimientos por } \\
\text { residente por mes }\end{array}$ & 9,6 & 35 & 15,7 & 28,6 & 15,7 \\
\hline
\end{tabular}

Tabla 2. Número y porcentaje de procedimientos por rotación.

\begin{tabular}{lcc}
\hline \multicolumn{1}{c}{ Rotación } & $\begin{array}{c}\text { Número de } \\
\text { procedimientos }\end{array}$ & $\%$ \\
\hline Cirugía general & 15247 & 65,7 \\
Cirugía de cabeza y cuello & 1125 & 4,8 \\
Cirugía pediátrica & 888 & 3,8 \\
Cirugía vascular & 737 & 3,2 \\
Cirugía laparoscópica & 732 & 3,2 \\
Cirugía de colon y recto & 581 & 2,5 \\
Cirugía de trauma & 444 & 1,9 \\
Cirugía hepatobiliar y trasplantes & 441 & 1,9 \\
Cirugía de mama y tejidos blandos & 354 & 1,5 \\
Cirugía de tórax & 348 & 1,5 \\
Demás rotaciones (Cirugía & & \\
Cardiovascular, Gastrointestinal, & & \\
Oncológica, Plástica, & 2309 & 10,0 \\
Gastroenterología, Cuidados & & \\
intensivos, Bariátrica, Urología) & & \\
\hline
\end{tabular}

(77,5 \%), apendicectomía (33,5 \%) y herniorrafia inguinal $(16,1 \%)$ El listado de los procedimientos y diagnósticos más frecuentes se presenta en las tablas 3 y 4 .

La distribución de los procedimientos por año de residencia fue la siguiente: Primer año 6181 (20,9\%), segundo año 9570 (32,3\%), tercer año $7980(26,9 \%)$ y cuarto año 5891 (19,9\%). El rol del residente durante el primer año de residencia fue de primer ayudante en el $55 \%$ de los procedimientos, y a partir del segundo año, el rol predominante fue cirujano principal $(58,3 \%$ segundo año, 63,8 \% tercer año y $72,7 \%$ en cuarto año) (figura 1). En el 70,9 \% de los procedimientos los residentes autoevaluaron su desempeño como "excelente" (figura 2).

Con la información disponible, se infirió cómo sería la formación del residente promedio año por año. Así, al finalizar la residencia, la mediana de procedimientos sería de 1297 registros (tabla 5). 
Tabla 3. Procedimientos más frecuentes.

\begin{tabular}{|c|c|c|}
\hline Procedimiento & Número & $\%$ \\
\hline Colecistectomía & 4341 & 14,7 \\
\hline Apendicectomía & 2558 & 8,6 \\
\hline Herniorrafia inguinal & 2059 & 7,0 \\
\hline Herniorrafia umbilical & 1225 & 4,1 \\
\hline Lavado peritoneal & 1198 & 4,0 \\
\hline Laparotomía exploratoria & 970 & 3,3 \\
\hline Laparoscopia diagnóstica & 816 & 2,8 \\
\hline Toracostomía & 725 & 2,4 \\
\hline Tiroidectomía total & 642 & 2,2 \\
\hline Safenectomía & 587 & 2,0 \\
\hline Varicectomía & 516 & 1,7 \\
\hline Eventrorrafia simple & 448 & 1,5 \\
\hline Catéter venoso implantable & 434 & 1,5 \\
\hline $\begin{array}{l}\text { Drenaje de absceso de tejidos } \\
\text { blandos }\end{array}$ & 394 & 1,3 \\
\hline Resección lipoma & 394 & 1,3 \\
\hline Liberación de adherencias & 348 & 1,2 \\
\hline $\begin{array}{l}\text { Catéter venoso central } \\
\text { subclavio }\end{array}$ & 339 & 1,1 \\
\hline Vaciamiento central de cuello & 326 & 1,1 \\
\hline Traqueostomía & 285 & 0,9 \\
\hline $\begin{array}{l}\text { Anastomosis de intestino } \\
\text { delgado }\end{array}$ & 267 & 0,9 \\
\hline
\end{tabular}

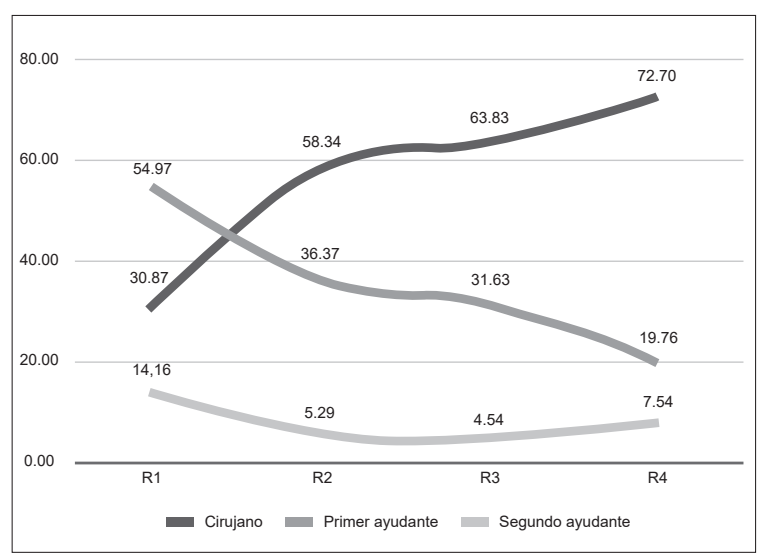

Gráfica 1. Rol del residente según año de residencia
Tabla 4. Diagnósticos más frecuentes (CIE-10)

\begin{tabular}{|c|c|c|}
\hline Diagnóstico & Número & $\%$ \\
\hline Apendicitis aguda, no especificada & 1993 & 8,6 \\
\hline $\begin{array}{l}\text { Cálculo de la vesícula biliar con } \\
\text { colecistitis aguda }\end{array}$ & 1594 & 6,9 \\
\hline $\begin{array}{l}\text { Hernia inguinal unilateral o no } \\
\text { especificada, sin obstrucción ni gangrena }\end{array}$ & 1421 & 6,1 \\
\hline Otras colelitiasis & 1166 & 5,0 \\
\hline $\begin{array}{l}\text { Hernia umbilical sin obstrucción ni } \\
\text { gangrena }\end{array}$ & 1092 & 4,7 \\
\hline Cálculo de la vesícula biliar sin colecistitis & 800 & 3,4 \\
\hline Tumor maligno de la glándula tiroides & 670 & 2,9 \\
\hline $\begin{array}{l}\text { Hernia ventral sin obstrucción ni } \\
\text { gangrena }\end{array}$ & 565 & 2,4 \\
\hline Insuficiencia venosa (crónica) (periférica) & 487 & 2,1 \\
\hline $\begin{array}{l}\text { Hernia inguinal bilateral, sin obstrucción } \\
\text { ni gangrena }\end{array}$ & 447 & 1,9 \\
\hline Colecistitis aguda & 389 & 1,7 \\
\hline $\begin{array}{l}\text { Tumor maligno de la mama, parte no } \\
\text { especificada }\end{array}$ & 312 & 1,3 \\
\hline Peritonitis aguda & 278 & 1,2 \\
\hline $\begin{array}{l}\text { Cálculo de la vesícula biliar con otra } \\
\text { colecistitis }\end{array}$ & 258 & 1,1 \\
\hline Apendicitis aguda con absceso peritoneal & 242 & 1,0 \\
\hline Otras peritonitis & 235 & 1,0 \\
\hline Fístula del intestino & 227 & 1,0 \\
\hline $\begin{array}{l}\text { Tumor benigno lipomatoso de piel y del } \\
\text { tejido subcutáneo del tronco }\end{array}$ & 209 & 0,9 \\
\hline $\begin{array}{l}\text { Apendicitis aguda con peritonitis } \\
\text { generalizada }\end{array}$ & 197 & 0,8 \\
\hline Tumor maligno del recto & 193 & 0,8 \\
\hline Tumor maligno del colon ascendente & 175 & 0,8 \\
\hline
\end{tabular}

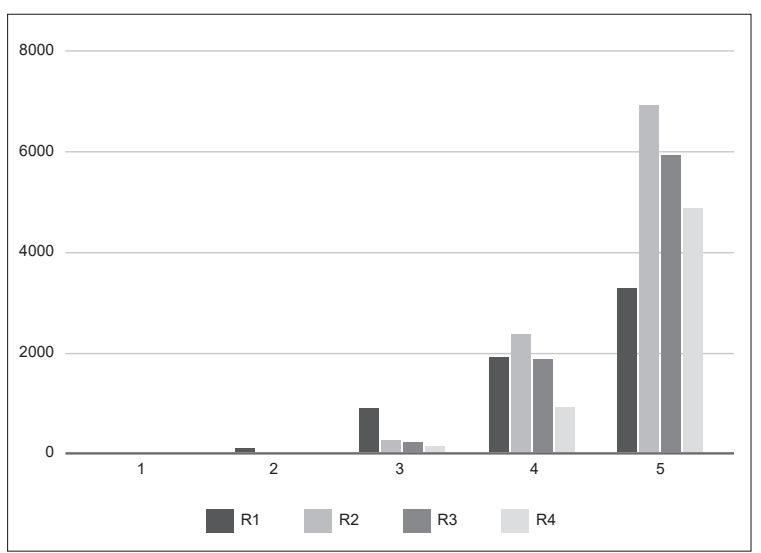

Gráfica 2. Autoevaluación según año de residencia. 
Tabla 5. Distribución de procedimientos por año de residencia.

\begin{tabular}{lcc}
\hline $\begin{array}{c}\text { Año de } \\
\text { residencia }\end{array}$ & $\begin{array}{c}\text { Promedio } \\
\text { entradas / mes }\end{array}$ & $\begin{array}{c}\text { Promedio } \\
\text { entradas / año }\end{array}$ \\
\hline Primer año & 23,4 & 280,8 \\
Segundo año & 23,3 & 279,5 \\
Tercer año & 36,6 & 439,2 \\
Cuarto año & 24,8 & 298,2 \\
Total & & 1297,7 \\
\hline
\end{tabular}

\section{Discusión}

Los hallazgos principales de este estudio fueron:

1. En promedio, cada residente realizó 27 cirugías por mes; más del $50 \%$ de estos procedimientos fueron electivos y se realizaron en rotaciones de cirugía general;

2. Los principales procedimientos en los que participaron los residentes fueron: colecistectomía, apendicectomía, reparo de hernias de la pared abdominal y lavado peritoneal (estos procedimientos se realizaron predominantemente por vía abierta, con excepción de la colecistectomía);

3. En estos procedimientos, el rol predominante del residente fue de cirujano principal desde momentos tempranos en su formación (a partir del segundo año).

Existen varias explicaciones para el primer hallazgo. Con una mediana de 306,6 registros al año, un residente se graduaría con 1226 registros al final de su entrenamiento. Estos datos, se asemejan a lo reportado en la literatura. Es importante recordar que la experiencia y exposición quirúrgica puede variar incluso dentro de varios programas en una misma ciudad, dependiendo de los lugares de rotación, enfoque del currículum de la universidad, forma de medición, entre otros ${ }^{1,9,13,15,16}$. A nivel nacional, Niño evaluó las bitácoras de treinta y siete residentes de la Universidad del Rosario durante un periodo de 11 años y encontró, con una significativa variabilidad, un promedio de $496(+230)$ cirugías anuales en su programa ${ }^{13}$. Siendo el presente estudio un esfuerzo conjunto, llevado a cabo en 3 ciudades del país, esta información es una adición importante al agregado de datos existentes.

Por otra parte, en dos reportes españoles sobre el "libro informático del residente quirúrgico", Gómez-Diaz y Serra-Aracil describen un promedio de 250 y 265 cirugías por año de residencia en España, respectivamente ${ }^{17,18}$. Por su parte, Elsey et al, realizaron una revisión detallada de la experiencia quirúrgica al finalizar la residencia a nivel mundial y analizaron las diferencias entre países ${ }^{9}$. Entre sus hallazgos más relevantes describen una escasez de datos en países diferentes a Estados Unidos, por la marcada variabilidad en las cirugías totales realizadas al final de la residencia y por la falta de acuerdo en el número mínimo de cirugías exigidos en los programas. En Estados Unidos, por ejemplo, se reporta un rango de 600-2785 procedimientos por residente, mientras que en Reino Unido es de 783-3764 (al terminar la residencia).

El número promedio de cirugías por año de residencia en los países evaluados en el meta análisis fue de 307 y 319 en dos estudios en Gran Bretaña, 232 en Holanda, 183 en Estados Unidos y 196 en Tailandia. Es de suma importancia tener en cuenta que la duración de la residencia es diferente en cada uno de estos países. Su meta análisis arrojó un estimado de 1366 (IC 95\% $_{1026-1707)}$ cirugías por residente al completar su residencia, con una heterogeneidad significativa (I2 99,6\%).

Varios países cuentan con mínimos de cirugías como criterios de evaluación, promoción y graduación. Entre ellos se destacan los ejemplos de Estados Unidos (mínimo de 850 cirugías, con 200 de ellas en el último año) ${ }^{19}$, Reino Unido (1600 cirugías) ${ }^{9}$ y España (423 cirugías como cirujano principal) ${ }^{18}$. En Chile, por ejemplo, la Sociedad de Cirujanos de Chile, sugiere para sus programas al menos 300 operaciones mayores como cirujano y otras 400 como asistente ${ }^{20}$. En el país, el número mínimo de procedimientos no es un requisito de grado ni un medidor de calidad. Con la información aquí presentada, no es posible aun sugerir un número mínimo esperado y hace 
falta realizar una recolección de información más prolongada y un análisis más profundo de estos resultados.

$\mathrm{Al}$ analizar los otros resultados evidenciamos que los residentes se exponen, casi en igual proporción, a la cirugía urgente y electiva, y con muy poca frecuencia a la cirugía emergente. La rotación de cirugía general aporta la gran mayoría de casos registrados y posiblemente tenga que ver con el porcentaje mayoritario destinado a esta rotación durante los programas de residencias en Colombia. Las rotaciones de segunda especialidad tienen una distribución homogénea de cantidad de registros, todos muy inferiores a la rotación de cirugía general, posiblemente por la complejidad de los procedimientos, la presencia de Fellows en las rotaciones y el tiempo más corto para estas rotaciones en el curriculum del posgrado.

Con respecto al segundo hallazgo, las cirugías más frecuentemente realizadas reflejan lo que posiblemente sea el día a día de un cirujano general en el país y podrían definitivamente incluirse como cirugías "trazadoras" o "índice" durante la formación quirúrgica, para poder tener un seguimiento sobre la participación de los residentes en estos procedimientos. La vía mínimamente invasiva se reportó en el 22,6 \% de las cirugías totales, cifra mayor al $16 \%$ reportado en la experiencia española ${ }^{18}$. Hay que aclarar que este dato se toma del total de procedimientos disponibles. Al reducir este listado a los procedimientos en abdomen y más aún por procedimientos más frecuentes, vemos un porcentaje importante en su uso, acorde con la tendencia actual respecto a estándares de manejo en las enfermedades altamente prevalentes, tanto urgentes (apendicitis, colecistitis) como electivas (colelitiasis, hernias inguinales).

Respecto al último hallazgo, relacionado con la autonomía, es importante mencionar que los residentes de los programas evaluados desempeñaron un rol principal en más de la mitad de los casos reportados en sus registros, actuando como "cirujano" en un número elevado de pacientes. Este rol es cada vez más protagónico, conforme avanza el residente en su nivel de formación.
No obstante, el papel que un residente asigna a su participación en un procedimiento no mide por completo la competencia para llevar a cabo el mismo. Como se mencionó antes, el cumplimiento de un número mínimo de procedimientos no asegura que un residente esté capacitado para realizarlo de manera independiente una vez graduado. Existe un constante interés por mejorar las deficiencias actuales en autonomía y confianza para lograr una práctica quirúrgica independiente segura. Un adecuado balance entre autonomía y supervisión, a medida que avanza el entrenamiento, tiene efectos positivos sobre el desempeño académico, profesional y clínico del residente. En 2018, Domínguez et al, compararon las percepciones, entre cirujanos y residentes, en autonomía y supervisión, para algunos procedimientos quirúrgicos. Pese a que existía congruencia en la mayoría de ellos, en algunos de los procedimientos más frecuentes, se presentaron diferencias estadísticamente significativas, atribuidas entre otras a técnicas novedosas, pacientes sometidos a cirugías complejas y políticas institucionales que llevan a que el residente finalmente no logre la autonomía esperada bajo una menor supervisión ${ }^{1}$. Dicho esto, es valioso a futuro agregar el seguimiento de retroalimentación del profesor a los registros del residente, para lograr una evaluación más objetiva de su desempeño.

\section{Fortalezas y limitaciones}

Este estudio cuenta con varias fortalezas, como la participación de varios programas de cirugía de diferentes regiones del país, el uso de una herramienta estandarizada (Bitácora Logbook), que favorece las comparaciones entre programas e individuos y es personalizable a cada programa según sus listados de profesores, hospitales y rotaciones y, finalmente, la adecuada adherencia de los médicos residentes, con registros durante los 12 meses del estudio.

No obstante, el estudio también posee limitaciones. Una de estas es la veracidad del dato, que en la mayoría de casos depende del residente, ya que cada registro no es directamente validado por un supervisor (este es un problema común en la 
mayoría de bitácoras). Igualmente, las estadísticas mostradas dependen exclusivamente de un correcto diligenciamiento de la bitácora electrónica. Por último, el periodo de evaluación es corto (12 meses) y la autoevaluación es subjetiva y no involucra retroalimentación del docente

\section{Implicaciones para la práctica y oportunidades de investigación futura}

Para un futuro, esperamos poder aumentar las universidades participantes y lograr un periodo de tiempo mayor. Asimismo, es ideal involucrar al cirujano supervisor, de manera que este logre revisar la información de los registros y optimizar la medida de autoevaluación del desempeño del residente, según escalas validadas para esto. De ser posible y conociendo experiencias de otros países, lograr acoplar a la bitácora, no sólo su actividad quirúrgica, sino la producción y participación académica e investigativa, que permita conocer un perfil más integral del residente.

\section{Conclusiones}

Es posible lograr un registro de bitácora a nivel nacional. Para esto es importante la participación de los jefes de programas de Cirugía general y los médicos residentes, así como el uso de una herramienta amigable y moldeable a las necesidades. Los residentes colombianos realizan un número de cirugías similares o mayores a lo descrito en otros países y cumplen un papel protagónico en la mayoría de ellas. La estadística afirma que debemos actualizar nuestras expectativas según la formación que reciben actualmente los residentes.

\section{Consideraciones éticas}

Consentimiento informado: El trabajo no involucró experimientos con seres humanos y se observaron las normas de la Declaración de Helsinki de 1975, por lo que no se requirió diligenciamiento de consentimiento informado por parte de los pacientes.

Conflicto de intereses: los autores declaran no tener ningún conflicto de interés.

Fuente de financiación: recursos propios de los autores.

\section{Contribución de los autores}

- Concepción y diseño del estudio: Sebastian Sierra, Luis Carlos Domínguez, Lilian Torregrosa.

- Adquisición de datos: Sebastian Sierra.

- Análisis e interpretación de datos: Sebastian Sierra, Luis Carlos Domínguez, Lilian Torregrosa.

- Redacción del manuscrito: Sebastian Sierra, Luis Carlos Domínguez, Lilian Torregrosa.

- Revisión crítica: Sebastian Sierra, Luis Carlos Domínguez, Lilian Torregrosa.

\section{Referencias}

1. Domínguez LC, Urueña N, Sanabria Á, Pepín JJ, Mosquera M, Vega V, Osorio C. Autonomía y supervisión del residente de cirugía: ¿se cumplen las expectativas en el quirófano? Educ Médica. 2018;19:208-16.

2. Harrington CM, Kavanagh DO, Ryan D, Dicker P, Lonergan PE, Traynor O, Tierney S. Objective scoring of an electronic surgical logbook: Analysis of impact and observations within a surgical training body. Am J Surg. 2017;214:962-8.

https://doi.org/10.1016/j.amjsurg.2017.07.028

3. Safavi A, Lai S, Butterworth S, Hameed M, Schiller D, Skarsgard E. Does operative experience during residency correlate with reported competency of recent general surgery graduates? Can J Surg. 2012;55:S171S177. https://doi.org/10.1503/cjs.020811

4. Fronza JS, Prystowsky JP, DaRosa D, Fryer JP. Surgical residents' perception of competence and relevance of the clinical curriculum to future practice. J Surg Educ. 2012;69:792-7. https://doi.org/10.1016/j.jsurg.2012.05.014

5. Potts JR. Assessment of Competence: The Accreditation Council for Graduate Medical Education/Residency Review Committee Perspective. Surg Clin North Am. 2016;96:15-24.

https://doi.org/10.1016/j.suc.2015.08.008

6. Comisión para la Transformación de la Educación Médica en Colombia. Documento de Recomendaciones para la Transformación de la Educación Médica en Colombia. Disponible en: https://www.minsalud.gov.co/sites/ rid/Lists/BibliotecaDigital/RIDE/VS/MET/recomendaciones-comision-para-la-transformacion.pdf

7. Morán-Barrios J. La evaluación del desempeño o de las competencias en la práctica clínica. 1.a Parte: principios y métodos, ventajas y desventajas. Educ Médica. 2016;17:130-9.

https://doi.org/10.1016/j.edumed.2016.07.001

8. Harrington CM, Jang SS, Mangaoang D, O’Flynn E, Minja C, Chikoya L, et al. Integration and sustainability of electronic surgical Logbooks in Sub-Saharan Africa. World J Surg. 2020;44:3259-67. https://doi.org/10.1007/s00268-020-05613-z

9. Elsey EJ, Griffiths G, Humes DJ, West J. Meta-analysis of operative experiences of general surgery trainees 
during training. Br J Surg. 2017;104:22-33. https://doi.org/10.1002/bjs.10396

10. Brasel KJ, Klingensmith ME, Englander R, Grambau M, Buyske J, Sarosi G, Minter R. Entrustable professional activities in general surgery: development and implementation. J Surg Educ. 2019;76:1174-86. https://doi.org/10.1016/j.jsurg.2019.04.003

11. ICFES - ASCOFAME. Cirugía General. Bogotá, D.C. Fecha de consulta: 25 de mayo de 2020. Disponible en: http://ascofame.org.co/web/wp-content/uploads/ 2016/08/CirugaGeneral.

12. Bermúdez C, Monroy A, Torregrosa L, Henao F. Estado actual de la formación de residentes de cirugía general en Colombia. Rev Colomb Cir. 2006;21:225-39.

13. Niño L, García MXC, Navas GA, Daza LAM. Experiencia Quirúrgica de los Residentes de Cirugía General de la Universidad del Rosario 2006-2017. 2018. Disponible en: https://repository.urosario.edu.co/handle/10336/14414

14. Sanabria ÁE, Contreras MJ, Aristizábal Giraldo H. Experiencia quirúrgica de la Sección de Cirugía General. Rev Colomb Cir. 2002;17:11.

15. Targarona-Soler EM, Jover-Navalon JM, Gutierrez-Saiz J, Turrado-Rodríguez V, Parrilla-Paricio P. ¿Qué operan los residentes españoles durante la residencia?: análisis de la aplicabilidad del programa de la especialidad de Cirugía General y Digestiva al finalizar el periodo de residencia. Cir Esp. 2015;93:152-8. https://doi.org/10.1016/j.ciresp.2015.01.001
16. Webber EM, Ronson AR, Gorman LJ, Taber SA, Harris KA. The Future of General Surgery: Evolving to meet a changing practice. J Surg Educ. 2016;73:496-503. https://doi.org/10.1016/j.jsurg.2015.12.002

17. Gómez-Díaz CJ, Luna-Aufroy A, Rebasa-Cladera P, Serra-Pla S, Jurado-Ruiz C, Mora-López L, et al. Libro informático del residente de cirugía: Un paso adelante. Cir Esp. 2015;93:651-7. https://doi.org/10.1016/j.cireng.2015.11.001

18. Serra-Aracil X, Navarro-Soto S, Hermoso-Bosch J, Miguelena JM, Ramos JL, Martín-Pérez E, et al. Estudio prospectivo, multicéntrico sobre la actividad de los residentes de cirugía general y del aparato digestivo en España a través del libro informático del residente. Cir Esp. 2012;90:518-24.

https://doi.org/10.1016/j.ciresp.2012.05.014

19. The American Board of Surgery. Booklet of information Surgery 2019-2020. Philadelphia: American Board of Surgery Inc. Disponible en:

https://www.absurgery.org/xfer/BookletofInfo-Surgery.pdf

20. Hepp J, Csendes A, Ibáñez F, Llanos O, San Martín S. Programa de la especialidad Cirugía General. Definiciones y propuestas de la Sociedad de Cirujanos de Chile. Rev Chil Cir. 2008;60:79-85. http://dx.doi.org/10.4067/S0718-40262008000100017 